\title{
Polymorphisms of rs2483205 and rs562556 in Proprotein Convertase Subtilisin/Kexin Type 9 (PCSK9) Gene are Associated With Coronary Artery Disease
}

\section{Min-Tao Gai}

Xinjiang Key Laboratory of Cardiovascular Disease, Clinical Medical Research Institute, First Affiliated Hospital of Xinjiang Medical University

\section{Dilare Adi}

Department of Cardiology, First Affiliated Hospital of Xinjiang Medical University

\section{Xiao-Cui Chen}

Xinjiang Key Laboratory of Cardiovascular Disease, Clinical Medical Research Institute, First Affiliated Hospital of Xinjiang Medical University

Fen Liu

Xinjiang Key Laboratory of Cardiovascular Disease, Clinical Medical Research Institute, First Affiliated Hospital of Xinjiang Medical University

\section{Xiang Xie}

Department of Cardiology, First Affiliated Hospital of Xinjiang Medical University

\section{Yi-Ning Yang}

Department of Cardiology, First Affiliated Hospital of Xinjiang Medical University

\section{Xiao-Ming Gao}

Xinjiang Key Laboratory of Cardiovascular Disease, Clinical Medical Research Institute, First Affiliated Hospital of Xinjiang Medical University

\section{Xiang Ma}

Department of Cardiology, First Affiliated Hospital of Xinjiang Medical University

\section{Zhen-Yan Fu}

Department of Cardiology, First Affiliated Hospital of Xinjiang Medical University

\section{Yi-Tong Ma}

Department of Cardiology, First Affiliated Hospital of Xinjiang Medical University

\section{Bang-dang Chen ( $\sim$ chenbangdang@126.com )}

Xinjiang Key Laboratory of Cardiovascular Disease, Clinical Medical Research Institute, First Affiliated Hospital of Xinjiang Medical University 
Keywords: PCSK9, TT genotype, SNPs, lipid metabolism, CAD

Posted Date: January 22nd, 2021

DOI: https://doi.org/10.21203/rs.3.rs-141256/v1

License: (c) (1) This work is licensed under a Creative Commons Attribution 4.0 International License. Read Full License 


\section{Abstract}

PCSK9 plays a pivotal role in lipid metabolism. The present study is to explore the potential novel single nucleotide polymorphisms (SNPs) of the PCSK9 gene which is associated with coronary artery disease (CAD) in the Han population who lived in Xinjiang, China. We conducted a case-control study and genotyped four tag SNPs of the PCSK9 gene in 950 CAD and 1082 health subjects in the Han population. This study showed that for rs2483205, the distributions in genotypes had significant differences between the CAD and control groups $(p=0.025)$, and the subjects with TT genotype had lower total cholesterol (TC) level than CT genotype $(p=0.025)$. For rs562556, there were significant differences between the CAD and control groups for distributions of genotypes $(p=0.020)$, and the individuals with a $\mathrm{G}$ allele had a lower low-density lipoprotein cholesterol (LDL-C) level than an A allele $(p=0.048)$. Furthermore, the TT genotype of rs 2483205 and GG genotype of rs 562556 were independently associated with CAD (odds ratio $[\mathrm{OR}]=0.53,95 \%$ confidence interval $[\mathrm{Cl}]=0.29-0.95, p=0.032$ and $\mathrm{OR}=0.57,95 \% \mathrm{Cl}=0.34-0.95, p=$ 0.032 , respectively). In conclusion, the rs 2483205 and rs 562556 were significantly associated with CAD.

\section{Introduction}

CAD is the main cause of death worldwide. An epidemic study predicts that the prevalence of CAD has been increased rapidly at least until $20300^{1,2}$ Gene and environment are the main factors to affect the development of CAD, and $40 \%-60 \%$ is attributed to genetic factors. ${ }^{3}$ Moreover, genes are involved in lipid metabolism and it usually have a significant effect on CAD. ${ }^{4}$

The human PCSK9 gene, located on chromosome 1p32.3, was mainly expressed as an amino acid glycoprotein. As studies reported, PCSK9 has effects on lipid metabolism mainly through the degradation of the LDL receptors. ${ }^{5}$ The specific mechanisms are as follow, in intracellular, the PCSK9 was binding to the LDL receptor to promoted the lysosomal degradation of the receptor, ${ }^{6,7}$ meanwhile in extracellular, PCSK9 in circulating is conjoined the EGF-A domain of LDL receptors to prevent the LDL receptors from recycling to the cell surface. ${ }^{7}$ Dozens of studies have indicated that the PCSK9 genetic mutations were associated with CAD, and more than 50 functional PCSK9 genetic mutations affected cholesterol levels in plasma. 8,9 The individuals with a loss-of-function (LOF) mutation of PCSK9 probably presented as a lifelong low total cholesterol (TC) and LDL-C levels, and notably reduced incidence of CAD risk. On the contrary, the individuals with a gain-of-function (GOF) mutation probably manifested hypercholesterolemia and susceptibility to CAD. ${ }^{10,11}$ According to studies, there were $2.6 \%$ of nonsense mutations in PCSK9 that are responsible for a $28 \%$ decrease of LDL-C level and $88 \%$ decrease in the risk of CAD. ${ }^{11}$ Some missense mutations of the PCSK9 gene have also a notable effect on plasma LDL-C levels and usually cause mild hypocholesterolemia to protect against CAD. ${ }^{8}$

Polymorphic sites of the PCSK9 gene are various in different regions and races. The polymorphisms of the PCSK9 gene in the Han population living in the northwestern part of China have not ever been reported. Moreover, finding novel lipids related polymorphisms would contribute to finding potential 
therapeutic targets for hyperlipemia and CAD. Therefore, this study was to investigate whether the polymorphisms of the PCSK9 gene were associated with CAD in the Han population who lived in Xinjiang, China. Furthermore, we also explored the association between different genotypes of the polymorphisms in the PCSK9 gene and lipids levels in all subjects.

\section{Methods}

\section{Ethical approval of the study}

All subjects have permitted to proceed with DNA analysis, collect relevant clinical data, and sign informed consent. The Ethics Committee of the First Affiliated Hospital of Xinjiang Medical University has reviewed the operating protocol and approved this study. All procedures were accorded to the requirements of the Declaration of Helsinki.

\section{Subjects}

In this study, we included 950 cases and 1082 healthy controls in the Han population who lived in Xinjiang, China. This study has recruited the patients who underwent angiography examination and were diagnosed with CAD at the First Affiliated Hospital of Xinjiang Medical University from 2008 to 2015. The control groups were randomly selected with age-matched participants from the cardiovascular risk survey (CRS) study which has been reported previously. ${ }^{29,30}$ In brief, it was a cross-sectional study, which collected blood samples, demographic information, lifestyle data, and cardiovascular-related clinical characteristics from seven representative regions and multiethnic populations to explore the potential cardiovascular-related risk factors in the Xinjiang population of China. Individuals were excluded if they had a history of CAD. All the DNA samples of participants were extracted from the blood samples collected by EDTA contained tubes.

Inclusion criteria: all the patients occurred the symptom of typical chest pain, and they have performed the coronary angiography examination according to guidelines ${ }^{31}$. CAD was defined as the coronary angiography examined results demonstrated that at least one coronary artery stenosis $>50 \%$. Exclusion criteria: the patients accompanied with valvular heart disease, non-ischemic cardiomyopathy, heart failure, or congenital heart disease.

\section{Definition of cardiovascular risk factors}

The diagnosis standard of hypertension was that, according to the medical history and examination, individuals have been diagnosed with hypertension before, or at least 2 separate times examine results were showed the systolic blood pressure (SBP) $\geq 140 \mathrm{mmHg}$, or diastolic blood pressure (DBP) $\geq$ $90 \mathrm{mmHg}$ at sedentary state ${ }^{32}$. The diagnosis standard for diabetes was that the subjects with a diabetes history, or a glucose value $>11.1 \mathrm{mmol} / \mathrm{L}(200 \mathrm{mg} / \mathrm{dl})$ at any one time, or glucose level $>7.0 \mathrm{mmol} / \mathrm{L}$ $(126 \mathrm{mg} / \mathrm{dl})$ in fasting plasma on 2 separate occasions. Smoking is defined as the subjects declared regular tobacco use in 6 months 


\section{Routine blood test}

Using fresh collected fasting peripheral blood samples, we processed routine biochemical variables testing. Lipids parameters, including high-density lipoprotein (HDL), TC, triglyceride (TG), and LDL-C, were tested by the Dimension AR/AVL Clinical Chemistry System (DADE Bchring, Newark, NJ) in the Clinical Laboratory.

\section{Polymorphism Selection and Genotyping}

We selected four tag SNPs by screening from the International HapMap Project website database (https://www.hapmap.org) and Haploview 4.2 software. At last, rs11583680, rs2483205, rs2495477, and rs562556 were picked for the Chinese Han population. The cutoff of minor allele frequency (MAF) was set as $>0.05$, and linkage disequilibrium (LD) patterns with $r^{2}$ were set as $>0.8$. The $r s 11583680$, rs2483205, rs2495477, and rs562556 belonged to upstream transcript variant, intron variant, intron variant, and coding variant, respectively. The SNPs were using an improved multiplex ligation detection reaction (iMLDR) technique (Genesky Biotechnologies Inc., Shanghai, China) to genotype. A blinded fashion was applied in genotyping, which in the absence of information on the patients' clinical data. About $10 \%$ of the samples were genotyped twice to test the quality of genotyping results.

\section{Statistical analysis}

According to data categories, we arranged different present forms and analysis methods. Continuous variables with normal distribution were presented as mean \pm standard deviation (SD), and as the median in case of non-normal distribution. The difference between two groups was examined by the independent Student $t$-test in variables with normal distribution and was analyzed by the Mann-Whitney $U$ test when variables distributed as non-normally. The Chi-square test was applied to explore the differences in categorical variables. The independent association between polymorphisms and CAD was assessed by multiple logistic regression analysis. The Hardy-Weinberg equilibrium (HWE) was analyzed by the Chisquare test by separately calculated the frequencies of genotype in CAD and control subjects. Haplotype construction and LD test were conducted by the SHEsis software platform ${ }^{33}$. The $p$-value $<0.05$ represents statistical significance (2-tailed). SPSS version 22.0 software (SPSS, Inc., Chicago, IL) was used to perform all statistical analyses.

\section{Results}

\section{Clinical characteristics of participants}

There were 950 CAD subjects (mean age of $58.68 \pm 7.30$ and $47.14 \%$ of men) and 1082 controls (mean age of $58.68 \pm 7.29$ and $46.43 \%$ of men) that were involved in the analysis. As Table 1 shown, comparing the control groups, the clinical characteristics of CAD patients at baseline showed lower HDL, and higher body mass index (BMI), glucose, blood pressure, uric acid, TG, and prevalence of smoking, hypertension, 
alcohol intake, and diabetes (all $p<0.05$ ). However, the parameters of age, gender, LDL-C, and TC have not shown any differences between the groups.

\section{Distribution of genotypes in the polymorphisms of the PCSK9 gene between the CAD and control groups.}

Four SNPs of PCSK9 (rs11583680 C>T, rs2483205 C>T, rs2495477 A>G and rs562556 G>A) were genotyped in both CAD and control groups. As Table 2 exhibited, the distribution of each genotype, genetic models, and alleles of the four SNPs was separately examined in CAD patients and controls. Except for the rs2495477 in the CAD group, all the genotype frequencies in both groups were in HWE ( $p$ > 0.05). For the rs 2483205 , the distributions in CC, CT, and TT genotypes, and in its recessive model $\triangle T T$ vs. $\mathrm{CC}+\mathrm{CT} \otimes$ were different between the two groups ( $p=0.025$, and $p=0.008$, respectively). For the rs562556 , the distributions in $A A, A G$, and $G G$ genotype, $A$ and $G$ alleles, and its dominant model $\triangle A A$ vs. GG+AG were also significantly different between the two groups ( $p=0.020, p=0.005$ and $p=0.006$, respectively). However, comparing the control group, the distributions of genotypes, models, or alleles of rs 11583680 and rs2495477 have not shown any significant differences in the CAD and control groups $(p=0.294$, and $p=0.342$, respectively).

\section{Independent risk factors for CAD}

To determine whether the polymorphisms of the PCSK9 gene were the independent risk factors for CAD, we adjusted confounding risk factors, including BMI, TG, TC, HDL-C, and LDL-C, the prevalence of hypertension, diabetes, smoking, and drinking. We found that the recessive model (CC vs. TT+CT) of rs2483205 and the dominant model (AA vs. GG+AG) of rs562556 were still showed a significant association with CAD. The TT genotype of rs2483205 was indicated the protective effects on CAD (OR = $0.53,95 \% \mathrm{Cl}=0.29-0.95, p=0.032$ ) (Table 3 ), and the $\mathrm{GG}$ genotype of rs562556 was also exhibited a benefit effect $(\mathrm{OR}=0.57,95 \% \mathrm{Cl}=0.34-0.95, p=0.032)($ Table 4$)$.

\section{LD analysis}

Table 5 is displayed the patterns of LD analysis in the PCSK9 gene. We have identified that these four SNPs are located in the same haplotype block. Except for rs2483205 (SNP2) and rs2495477 (SNP3), all the $r^{2}$ values of SNPs were below 0.5 , which means that we could not construct haplotypes by SNP2 and SNP3 simultaneously. In addition, because the minor allele frequency (MAF) of SNP2 is larger than SNP3, we just used SNP1, SNP2, and SNP4 to construct the haplotypes. In further, because the |D'| for rs11583680 (SNP1)-SNP2, SNP1-SNP3, and SNP1-(rs562556) SNP4 were <0.5. So we do not use the SNP1 to construct haplotypes. At last, the SNP2 and SNP4 were used to construct haplotypes.

\section{Relationship between the haplotypes of the PCSK9 gene and CAD}

As Table 6 is shown, we established the haplotypes by combining the SNP2 and SNP4. Then, the distribution of the SNP2-SNP4 constructed haplotypes between the two groups was analyzed. The haplotype distributions of $\mathrm{C}-\mathrm{G}(\mathrm{H} 2)$ and $\mathrm{T}-\mathrm{G}(\mathrm{H} 4)$ were significantly different between the two groups 
$(p<0.05)$. The frequencies of $\mathrm{H} 2$ haplotype were significantly higher in the CAD group than in the control group $(\mathrm{OR}=1.97,95 \% \mathrm{Cl}: 1.013-3.842, p=0.042)$. However, compared with the healthy control subjects, the frequencies of $\mathrm{H} 4$ haplotype were significantly lower in patients with CAD (OR $=0.495,95 \% \mathrm{Cl}$ : 0.351 $0.699, p \otimes 0.001)$.

\section{The relationship between PSCK9 genotypes and lipids levels}

The relationship between the genotypes of polymorphisms and lipids levels was analyzed. Individuals with TT genotype in rs2483205 had significantly lower mean TC level than CT genotype $(4.62 \pm 2.62 \mathrm{mmol} / \mathrm{L}$ vs. $3.91 \pm 1.20 \mathrm{mmol} / \mathrm{L}, p=0.025)$, Figure $1 \mathrm{a}$. And there is lower mean LDL-C level in $\mathrm{G}$ allele than A allele in the rs562556 $(2.71 \pm 0.99 \mathrm{mmol} / \mathrm{L}$ vs. $2.41 \pm 0.97 \mathrm{mmol} / \mathrm{L}, p=0.048)$, Figure $1 \mathrm{~b}$. Nevertheless, neither HDL-C nor TG showed any significant difference among different genotypes of rs562556 or rs $2483205(p>0.05)$.

\section{Discussion}

In this study, we found that polymorphisms of rs562556 and rs2483205 in the PCSK9 gene associated with CAD, it was the first time reported in the Han population who lived in Xinjiang, China. The TT genotype of rs 2483205 polymorphism and GG genotype of rs562556 had protective effects in patients with CAD. Furthermore, we found that the mutations of rs2483205 and rs562556 polymorphisms exhibited reduced TC and LDL-C levels, respectively. Moreover, we revealed the individuals with H4 haplotype presented as a protective effect on CAD.

High LDL-C or TC concentrations have the causal association with increased cardiovascular risk. Multiple randomized controlled trials and meta-analyses indicated that reduced LDL-C levels were usually connected with a consistent and graded reduction in cardiovascular risk ${ }^{12-16}$. As a cornerstone and routine medicine for hyperlipidemia therapy, statins could effectively lower LDL-C most of the time, and are generally well tolerated. However, due to potential side effects, some individuals' poor response, and misinformation of statins therapy, many patients are reluctant in taking statins or adhere to treatment ${ }^{17,18}$. In recent years, PCSK9 has been regarded as a promising therapeutic target to regulate cholesterol metabolism. On lipid and metabolite profiles, metabolic effects of PCSK9 gene inhibitors are comparable with statin therapy ${ }^{18}$. In addition, studies indicated that combining PCSK9 inhibitors and statin can reduce LDL-C by $50-60 \%$, which is higher than statin therapy alone. Besides, in patients with atherosclerotic cardiovascular disease (ASCVD), PCSK9 inhibitors may reduce overall mortality and adverse cardiovascular events by combination with high-dose statins. Moreover, according to the 2019 guidelines for the management of dyslipidemias, PCSK9 inhibitors have been included in therapy for very high-risk ASCVD patients who would reach treatment targets on the maximum dose of ezetimibe and statin ${ }^{19,20}$. However, whether there are novel genetic targeted inhibitors of PCSK9 that could be applied to therapy ASCVD patients with fewer side effects and higher efficiency required further exploration. 
Population-based studies suggested that PCSK9 variants are associated with cholesterol levels and CAD in homozygous or compound heterozygous carriers ${ }^{8}$. Missense and nonsense mutations of PCSK9 probably result in hypocholesterolemia and have a protective effect on CAD. Previously, most studies about the PCSK9 gene were focused on several polymorphisms, such as E670G or R46L, which suggested that the PCSK9 gene was significantly associated with LDL-C levels and CAD ${ }^{21-23}$. Few studies explored the relationship between the rs562556 polymorphism and CAD. Two published studies have evaluated the associations between the rs 562556 polymorphism and high levels of lipids in patients with hypercholesterolemia and polycystic ovary syndrome, respectively ${ }^{24,25}$. Currently, a meta-analysis study proceeded with a pooled analysis and concluded that the $\mathrm{G}$ carriers of rs 562556 polymorphism had lower TC, LDL-C levels, and relative risk than the non-carriers ${ }^{26}$. These findings are in agreement with our results. In the present study, we also found that the $\mathrm{G}$ carriers of rs 562556 polymorphism were associated with lower LDL-C levels. Furthermore, several studies also explored the relationship between rs 562556 polymorphism and different diseases. A published study showed that the rs 562556 polymorphism had no association with myocardial infarction ${ }^{27}$. A clinical study indicated the mutations of the rs 562556 polymorphism were related to incidence of the carotid arterial plaques and elevated PCSK9 level ${ }^{28}$. Here, the present study for the first time uncovered that the GG genotype of 562556 polymorphism plays a protective role in CAD in the Han population. Meanwhile, few studies about rs2483205 polymorphism were reported. The protective effects of the TT genotype in rs2483205 polymorphism for CAD might be attributed to its lower TC level which proved in the present study. However, as a nonsense mutation, the specific mechanisms of how the rs2483205 polymorphism affects the TC level and the effects of rs2483205 on prognosis in patients with CAD were not clear.

The present studies have several limitations. First of all, this is a case-control study, which provides less information about the relationship between PCSK9 polymorphisms and long-term prognosis or adverse cardiovascular events. Second, the sample size is still small in this study. A large sample size prospective cohort study is required to further determine the relationship between PCSK9 polymorphisms and CAD disease and its prognosis. Third, the specific mechanisms of how rs562556 or rs2483205 influencing cholesterol metabolism and lowering the risk of CAD is still needing further research.

In conclusion, we for the first time proved that the polymorphisms of rs562556 and rs2483205 were associated with CAD in the Han population who lived in Xinjiang, China. The TT genotype of rs2483205, GG genotype of rs562556, and H4 haplotype had protective effects on CAD. In addition, mutations of rs2483205 and rs562556 polymorphisms were associated with lower levels of TC and LDL-C, respectively. The polymorphisms of rs562556 and rs2483205 in the PCSK9 gene would be a potential therapeutic target for the treatment of CAD by reducing lipid levels.

\section{Declarations}

\section{Acknowledgements}


This work was supported by the open project of Key Laboratory (No. 2017D04001) from Science and Technology Department of Xinjiang Uygur Autonomous Region.

\section{Author contributions}

Y.T.M. and B.D.C. conceived the present study; M.T.G. and D.A. designed the experiments and wrote the draft of the manuscript; Y.N.Y., G.X.M., Y.T.M. and B.D.C. gave the suggestion for this manuscript; X.C.C., X.M. and Z.Y.F. collected data; F.L., Y.N.Y. and X.X. performed statistical analyses; M.T.G., D.A., C.X.C., X.X. and X.M. collected samples and undertook laboratory experiments; Y.T.M. and B.D.C. supervised this study. All authors reviewed the manuscript.

\section{Competing interests}

The authors declare no competing interests.

\section{Additional information}

Correspondence and requests for materials should be addressed to B.D.C. and M.Y.T..

\section{References}

1. Mathers, C. D. \& Loncar, D. Projections of global mortality and burden of disease from 2002 to 2030. PLoS Med 3, e442, doi:10.1371/journal.pmed.0030442 (2006).

2. Benjamin, E. J. et al. Heart Disease and Stroke Statistics-2019 Update: A Report From the American Heart Association. Circulation 139, e56-e528, doi:10.1161/cir.0000000000000659 (2019).

3. Roberts, R. \& Stewart, A. F. Genes and coronary artery disease: where are we? J Am Coll Cardiol 60, 1715-1721, doi:10.1016/j.jacc.2011.12.062 (2012).

4. Luo, J., Yang, H. \& Song, B. L. Mechanisms and regulation of cholesterol homeostasis. Nat Rev Mol Cell Biol 21, 225-245, doi:10.1038/s41580-019-0190-7 (2020).

5. Lambert, G., Sjouke, B., Choque, B., Kastelein, J. J. \& Hovingh, G. K. The PCSK9 decade. J Lipid Res 53, 2515-2524, doi:10.1194/jlr.R026658 (2012).

6. Suzanne Benjannet, D. R., Rachid Essalmani, Janice Mayne, Louise Wickham. NARC-1/PCSK9 and its natural mutants: zymogen cleavage and effects on the low density lipoprotein (LDL) receptor and LDL cholesterol. J Biol Chem 279(47), 48865-48875, doi:10.1074/jbc.M409699200 (2004).

7. Da-Wei Zhang , T. A. L., Rita Garuti, Zhenze Zhao, Meghan McDonald. Binding of proprotein convertase subtilisin/kexin type 9 to epidermal growth factor-like repeat A of low density lipoprotein receptor decreases receptor recycling and increases degradation. J Biol Chem 282(25), 18602-18612, doi:10.1074/jbc.M702027200 (2007).

8. Abifadel, M. et al. Mutations and polymorphisms in the proprotein convertase subtilisin kexin 9 (PCSK9) gene in cholesterol metabolism and disease. Hum Mutat 30, 520-529, doi:10.1002/humu.20882 (2009). 
9. Davignon, J., Dubuc, G. \& Seidah, N. G. The influence of PCSK9 polymorphisms on serum low-density lipoprotein cholesterol and risk of atherosclerosis. Curr Atheroscler Rep 12, 308-315, doi:10.1007/s11883-010-0123-6 (2010).

10. Cohen, J. et al. Low LDL cholesterol in individuals of African descent resulting from frequent nonsense mutations in PCSK9. Nat Genet 37, 161-165, doi:10.1038/ng1509 (2005).

11. Cohen, J. C., Boerwinkle, E., Mosley, T. H., Jr. \& Hobbs, H. H. Sequence variations in PCSK9, low LDL, and protection against coronary heart disease. N Engl J Med 354, 1264-1272, doi:10.1056/NEJMoa054013 (2006).

12. Baigent, C. et al. Efficacy and safety of more intensive lowering of LDL cholesterol: a meta-analysis of data from 170,000 participants in 26 randomised trials. Lancet 376, 1670-1681, doi:10.1016/s0140-6736(10)61350-5 (2010).

13. Boekholdt, S. M. et al. Very low levels of atherogenic lipoproteins and the risk for cardiovascular events: a meta-analysis of statin trials. J Am Coll Cardio/ 64, 485-494, doi:10.1016/j.jacc.2014.02.615 (2014).

14. Brugts, J. J. et al. The benefits of statins in people without established cardiovascular disease but with cardiovascular risk factors: meta-analysis of randomised controlled trials. Bmj 338, b2376, doi:10.1136/bmj.b2376 (2009).

15. Fulcher, J. et al. Efficacy and safety of LDL-lowering therapy among men and women: meta-analysis of individual data from 174,000 participants in 27 randomised trials. Lancet 385, 1397-1405, doi:10.1016/s0140-6736(14)61368-4 (2015).

16. Mills, E. J. et al. Primary prevention of cardiovascular mortality and events with statin treatments: a network meta-analysis involving more than 65,000 patients. J Am Coll Cardio/ 52, 1769-1781, doi:10.1016/j.jacc.2008.08.039 (2008).

17. Adhyaru, B. B. \& Jacobson, T. A. Safety and efficacy of statin therapy. Nat Rev Cardio/ 15, 757-769, doi:10.1038/s41569-018-0098-5 (2018).

18. Hill, J. A. et al. Medical misinformation: vet the message! Eur Heart J Cardiovasc Pharmacother 5, 6263, doi:10.1093/ehjcvp/pvz001 (2019).

19. Gallego-Colon, D. A., Yosefy C. Statins and PCSK9 inhibitors: A new lipid-lowering therapy. Eur J Pharmacol 878, 173114, doi:10.1016/j.ejphar.2020.173114 (2020).

20. Allahyari A, J. T., Hagström E, Leosdottir M, Lundman P, Ueda P. Application of the 2019 ESC/EAS dyslipidaemia guidelines to nationwide data of patients with a recent myocardial infarction: a simulation study. Eur Heart J, ehaa034, doi:10.1093/eurheartj/ehaa034 (2020).

21. Adi D, X. X., Liu F,Yi-Tong Ma, Mayila Abudoukelimu , Yun Wu , Yong An. Relationships between genetic polymorphisms of E670G in PCSK9 gene and coronary artery disease: a meta-analysis. Int $J$ Clin Exp Med 8(8), 13251-13258 (2015).

22. Cai G, Z. B., Ganwei Shi , Weijin Weng, Chunyan Ma , Yanbin Song. The associations between proprotein convertase subtilisin/kexin type $9 \mathrm{E} 670 \mathrm{G}$ polymorphism and the risk of coronary artery 
disease and serum lipid levels: a meta-analysis. Lipids Health Dis 14:149, doi:10.1186/s12944-0150154-7 (2015).

23. Benn, M., Nordestgaard, B. G., Grande, P., Schnohr, P. \& Tybjaerg-Hansen, A. PCSK9 R46L, low-density lipoprotein cholesterol levels, and risk of ischemic heart disease: 3 independent studies and metaanalyses. Journal of the American College of Cardiology 55, 2833-2842, doi:10.1016/j.jacc.2010.02.044 (2010).

24. Anderson, J. M. et al. Influence of PCSK9 polymorphisms on plasma lipids and response to atorvastatin treatment in Brazilian subjects. J Clin Lipido/ 8, 256-264, doi:10.1016/j.jacl.2014.02.008 (2014).

25. Xavier, L. B. et al. Evaluation of PCSK9 levels and its genetic polymorphisms in women with polycystic ovary syndrome. Gene 644, 129-136, doi:10.1016/j.gene.2017.11.006 (2018).

26. Chuan, J., Qian, Z., Zhang, Y., Tong, R. \& Peng, M. The association of the PCSK9 rs562556 polymorphism with serum lipids level: a meta-analysis. Lipids Health Dis 18, 105, doi:10.1186/s12944-019-1036-1 (2019).

27. Shioji, K. et al. Genetic variants in PCSK9 affect the cholesterol level in Japanese. J Hum Genet 49, 109-114, doi:10.1007/s10038-003-0114-3 (2004).

28. Ferreira, J. P. et al. PCSK9 Protein and rs562556 Polymorphism Are Associated With Arterial Plaques in Healthy Middle-Aged Population: The STANISLAS Cohort. J Am Heart Assoc 9, e014758, doi:10.1161/jaha.119.014758 (2020).

29. Pan, S. et al. Appropriate body mass index and waist circumference cutoffs for categorization of overweight and central adiposity among Uighur adults in Xinjiang. PLoS One 8, e80185, doi:10.1371/journal.pone.0080185 (2013).

30. Luo, J. Y. et al. Prevalence, awareness, treatment and control of dyslipidemia among adults in northwestern China: the cardiovascular risk survey. Lipids Health Dis 13, 4, doi:10.1186/1476-511x13-4 (2014).

31. Gibbons, R. J. et al. ACC/AHA 2002 guideline update for the management of patients with chronic stable angina--summary article: a report of the American College of Cardiology/American Heart Association Task Force on practice guidelines (Committee on the Management of Patients With Chronic Stable Angina). J Am Coll Cardiol 41, 159-168, doi:10.1016/s0735-1097(02)02848-6 (2003).

32. 1999 World Health Organization-International Society of Hypertension Guidelines for the Management of Hypertension. Guidelines Subcommittee. J Hypertens 17, 151-183 (1999).

33. Li Z, Z. Z., He Z, Tang W, Li T, Zeng Z, He L, Shi Y. A partition-ligation-combination-subdivision EM algorithm for haplotype inference with multiallelic markers: update of the SHEsis (http://analysis.biox.cn). Cell Res 19(4), 519-523 (2009).

\section{Tables}

Table 1. Baseline characteristics of control subjects and patients with coronary heart disease 


\begin{tabular}{|c|c|c|c|}
\hline Characteristics & control[n=1082 & CAD $\square n=950 \square$ & $p$ value \\
\hline Age & $56.67 \pm 8.73$ & $58.68 \pm 7.29$ & 0.981 \\
\hline Male gender, n (\%) & 514(46.43区 & $436(47.14)$ & 0.746 \\
\hline Smoking, n (\%) & $327(30.31)$ & $453(47.62)$ & $<0.001 *$ \\
\hline Alcohol intake, n (\%) & $182(16.80)$ & $479(50.45)$ & $<0.001 *$ \\
\hline $\mathrm{BMI}, \mathrm{kg} / \mathrm{m} 2$ & $25.56 \pm 4.02$ & $26.37 \pm 3.46$ & $<0.001 *$ \\
\hline SBP, mmHg & $134.99 \pm 22.61$ & $150.50 \pm 31.83$ & $<0.001$ * \\
\hline $\mathrm{DBP}, \mathrm{mmHg}$ & $85.42 \pm 16.63$ & $92.10 \pm 20.46$ & $<0.001$ * \\
\hline Hypertension, n (\%) & $485(45.07)$ & $516(54.3)$ & $<0.001$ * \\
\hline Uric Acid, mmol/L & $270.74 \pm 85.22$ & $310.77 \pm 112.61$ & $<0.001$ * \\
\hline Glucose, $\mathrm{mmol} / \mathrm{L}$ & $5.30 \pm 1.90$ & $6.23 \pm 2.62$ & $<0.001$ * \\
\hline Diabetes, n (\%) & $88(8.13)$ & $233(24.53)$ & $<0.001$ * \\
\hline $\mathrm{TG}, \mathrm{mmol} / \mathrm{L}$ & $1.56 \pm 1.07$ & $2.03 \pm 1.33$ & $<0.001$ * \\
\hline $\mathrm{TC}, \mathrm{mmol} / \mathrm{L}$ & $4.27 \pm 0.93$ & $4.38 \pm 1.63$ & 0.058 \\
\hline HDL-C, mmol/L & $1.25 \pm 0.47$ & $0.98 \pm 0.40$ & $<0.001 *$ \\
\hline LDL-C, mmol/L & $2.66 \pm 0.69$ & $2.68 \pm 0.98$ & 0.591 \\
\hline
\end{tabular}

Abbreviations: BMI, body mass index; SBP, systolic blood pressure; DBP, diastolic blood pressure; TC, total cholesterol; TG, triglyceride; LDL-C, low density lipoprotein-cholesterol; HDL-C, high density lipoproteincholesterol. * compared with control group, $p$-values $<0.001$.

Table 2. Genotype and allele distributions in control subjects and patients with CAD 


\begin{tabular}{|c|c|c|c|c|c|}
\hline Variants & $\begin{array}{l}\text { Control, } n(\%) \\
(n=1082)\end{array}$ & $\begin{array}{l}\text { CAD, } \\
n(\%) \\
(n=950)\end{array}$ & $\begin{array}{l}\text { CAD } p \\
\text { Value* } \\
{ }^{*}(H-W)\end{array}$ & $\begin{array}{l}\text { Control } p \\
\text { Value* }(\mathrm{H}-\mathrm{W})\end{array}$ & $\begin{array}{l}\text { P- } \\
\text { Value } \oint\end{array}$ \\
\hline \multicolumn{6}{|c|}{ rs11583680(SNP1) } \\
\hline \multicolumn{6}{|c|}{ Genotyping } \\
\hline $\mathrm{CC}$ & $882(81.52)$ & $750(78.95)$ & & & \\
\hline CT & $187(17.28)$ & 184(19.37) & & & \\
\hline TT & $13(1.20)$ & $16(1.68)$ & & & 0.294 \\
\hline \multicolumn{6}{|c|}{ Dominant model } \\
\hline $\mathrm{CC}$ & $882(81.52)$ & $750(78.95)$ & & & \\
\hline $\mathrm{TT}+\mathrm{CT}$ & $200(18.48)$ & $200(21.05)$ & 0.231 & 0.389 & 0.146 \\
\hline \multicolumn{6}{|c|}{ Recessive model } \\
\hline TT & $13(1.20)$ & 16(1.68) & & & \\
\hline $\mathrm{CC}+\mathrm{CT}$ & 1069(98.80) & $934(98.32)$ & & & 0.360 \\
\hline \multicolumn{6}{|l|}{ Allele } \\
\hline C allele & 1951(90.16) & 1684(88.63) & & & \\
\hline T allele & $213(9.84)$ & $216(11.37)$ & & & 0.114 \\
\hline \multicolumn{6}{|c|}{ rs2483205(SNP2) } \\
\hline \multicolumn{6}{|c|}{ Genotyping } \\
\hline $\mathrm{CC}$ & $523(48.34)$ & $488(51.37)$ & & & \\
\hline CT & $456(42.14)$ & $402(42.32)$ & & & \\
\hline TT & $103(9.25)$ & $60(6.32)$ & & & $0.025 \oint$ \\
\hline \multicolumn{6}{|c|}{ Dominant model } \\
\hline $\mathrm{CC}$ & $523(48.34)$ & $488(51.37)$ & & & \\
\hline $\mathrm{TT}+\mathrm{CT}$ & $559(51.66)$ & $462(48.63)$ & 0.057 & 0.803 & 0.173 \\
\hline \multicolumn{6}{|c|}{ Recessive model } \\
\hline TT & $103(9.52)$ & $60(6.32)$ & & & \\
\hline $\mathrm{CC}+\mathrm{CT}$ & $979(90.48)$ & $890(93.68)$ & & & $0.008 \oint$ \\
\hline \multicolumn{6}{|l|}{ Allele } \\
\hline C allele & 1502(69.41) & 1298(71.32) & & & \\
\hline
\end{tabular}




\begin{tabular}{|c|c|c|c|c|}
\hline T allele & 662(30.69) & $522(28.78)$ & & 0.189 \\
\hline \multicolumn{5}{|c|}{ rs2495477(SNP3) } \\
\hline \multicolumn{5}{|c|}{ Genotyping } \\
\hline AA & $502(46.40)$ & $463(48.74)$ & & \\
\hline$A G$ & $486(44.92)$ & $419(44.11)$ & & \\
\hline $\mathrm{GG}$ & $94(8.69)$ & $68(7.16)$ & & 0.342 \\
\hline \multicolumn{5}{|c|}{ Dominant model } \\
\hline AA & $502(46.40)$ & 463(48.74) & & \\
\hline$G G+A G$ & $580(53.60)$ & $487(51.26)$ & $0.041^{*}$ & 0.292 \\
\hline \multicolumn{5}{|c|}{ Recessive model } \\
\hline GG & $94(8.69)$ & $68(7.16)$ & & \\
\hline$A A+A G$ & 988(91.31) & 882(92.84) & & 0.204 \\
\hline \multicolumn{5}{|l|}{ Allele } \\
\hline A allele & $1490(68.9)$ & $1345(70.79)$ & & \\
\hline G allele & 674(31.1) & $555(29.21)$ & & 0.180 \\
\hline \multicolumn{5}{|c|}{ rs562556(SNP4) } \\
\hline \multicolumn{5}{|c|}{ Genotyping } \\
\hline AA & $962(88.91)$ & 879(92.53) & & \\
\hline$A G$ & 118(10.91) & 70(7.37) & & \\
\hline GG & $2(0.18)$ & $1(0.11)$ & & $0.020 \oint$ \\
\hline \multicolumn{5}{|c|}{ Dominant model } \\
\hline AA & 962(88.91) & $879(92.53)$ & & \\
\hline$G G+A G$ & 120(11.09) & 71(7.47) & 0.746 & $0.005 \oint$ \\
\hline \multicolumn{5}{|c|}{ Recessive model } \\
\hline GG & $2(0.18)$ & $1(0.11)$ & & \\
\hline$A A+A G$ & 1080(99.82) & $949(99.89)$ & & 0.641 \\
\hline \multicolumn{5}{|l|}{ Allele } \\
\hline A allele & 2042(94.36) & 1828(96.21) & & \\
\hline G allele & $122(5.54)$ & 72(3.79) & & $0.006 \oint$ \\
\hline
\end{tabular}


Abbreviations: CAD, coronary artery disease.

* $p$ values $<0.05$ for Hardy-Weinberg equilibrium in CAD patients and controls.

$\oint p$ values $<0.05$ for distribution frequency for genotypes and alleles of the 4 SNPs in PCSK9 gene.

Table 3. Multiple logistic regression analysis for CAD patients and control subjects

\begin{tabular}{|lllll|}
\hline Risk factors & OR & $95 \% \mathrm{Cl}$ & wals & $\boldsymbol{p}$ \\
\hline rs2483205(TT vs. CC+CT) & 0.53 & $(0.29-0.95)$ & 4.60 & $0.032^{*}$ \\
\hline smoking & 1.43 & $(0.97-2.03)$ & 3.23 & 0.072 \\
\hline BMl, kg/m2 & 1.04 & $(0.99-1.08)$ & 2.89 & 0.069 \\
\hline Hypertension, n (\%) & 0.86 & $(0.62-1.20)$ & 0.78 & 0.377 \\
\hline Diabetes, n (\%) & 2.46 & $(1.29-4.68)$ & 7.46 & $0.006^{*}$ \\
\hline TG, mmol/L & 1.61 & $(1.36-1.92)$ & 29.21 & $<0.001^{*}$ \\
\hline HDL-C, mmol/L & 0.94 & $(0.89-1.00)$ & 3.74 & 0.053 \\
\hline TC, mmol/L & 0.81 & $(0.62-1.07)$ & 2.14 & 0.144 \\
\hline LDL, mmol/L & 1.40 & $(0.99-1.97)$ & 3.75 & 0.053 \\
\hline
\end{tabular}

Adjust: RS205RM, smoking, alcohol intake, hypertension, diabetes, TG, TC, HDL-C, LDL-C, and BMI. Abbreviations: BMI, body mass index; SBP, systolic blood pressure; DBP, Diastolic Blood Pressure; TC, total cholesterol; TG, triglyceride; LDL-C, low density lipoprotein-cholesterol; HDL-C, high density lipoprotein-cholesterol. *: $p$-values $<0.05$.

Table 4. Multiple Logistic Regression Analysis for CAD Patients and Control Subjects 


\begin{tabular}{|lllll|}
\hline Risk factors & OR & $95 \% \mathrm{Cl}$ & wals & $\boldsymbol{p}$ \\
\hline rs562556هGG+AG vs. AA囚 & 0.57 & $(0.34-0.95)$ & 4.58 & $0.032^{*}$ \\
\hline smoking & 1.40 & $(0.97-2.02)$ & 3.21 & 0.073 \\
\hline BMl, kg/m2 & 1.04 & $(0.99-1.09)$ & 3.27 & 0.071 \\
\hline Hypertension, n (\%) & 0.86 & $(0.62-1.20)$ & 0.77 & 0.380 \\
\hline Diabetes, n (\%) & 2.46 & $(1.29-4.69)$ & 7.43 & $0.006 *$ \\
\hline TG, mmol/L & 1.60 & $(1.35-1.90)$ & 28.75 & $<0.001 *$ \\
\hline HDL-C, mmol/L & 0.95 & $(0.89-1.01)$ & 3.26 & 0.071 \\
\hline TC, mmol/L & 0.81 & $(0.61-1.07)$ & 2.21 & 0.137 \\
\hline LDL, mmol/L & 1.40 & $(0.99-1.96)$ & 3.67 & 0.055 \\
\hline
\end{tabular}

Adjust: rs562556DM, smoking, alcohol intake, hypertension, Diabetes, TG, TC, HDL-C, LDL-C, and BMI. Abbreviations: BMI, body mass index; SBP, systolic blood pressure; DBP, Diastolic Blood Pressure; TC, total cholesterol; TG, triglyceride; LDL-C, low density lipoprotein-cholesterol; HDL-C, high density lipoprotein-cholesterol. *: $p$-values $<0.05$.

Table 5. Pairwise linkage disequilibrium for the four SNPs.

\begin{tabular}{|c|c|c|c|c|c|}
\hline & & \multicolumn{4}{|c|}{ D'values } \\
\hline & & SNP1 & SNP2 & SNP3 & SNP4 \\
\hline \multirow[t]{4}{*}{ r2 values } & SNP1 & & 0.44 & 0.41 & 0.08 \\
\hline & SNP2 & 0.06 & & 0.82 & 0.72 \\
\hline & SNP3 & 0.05 & 0.63 & & 0.78 \\
\hline & SNP4 & 0.00 & 0.06 & 0.07 & \\
\hline
\end{tabular}

$\left|D^{\prime}\right|$ above the diagonal and $r^{2}$ below the diagonal. The shadowed portion indicates $\left|D^{\prime}\right|<0.5$ and $r^{2}>0.5$ SNP1-4 = rs11583680, rs2483205, rs2495477, rs562556.

Table 6. Haplotype analysis in patients with CAD and control subjects. 


\begin{tabular}{|lllllll|}
\hline Haplotype & SNP2 & SNP4 & $\begin{array}{l}\text { Control, } n(\%) \\
(\mathbf{n = 1 0 8 2})\end{array}$ & $\begin{array}{l}\text { CAD, } n(\%) \\
(\mathbf{n = 9 5 0 )}\end{array}$ & OR[95\%Cl] & $P$ value \\
H1 & C & A & $1488.21(0.69)$ & $1354.26(0.71)$ & $\begin{array}{l}1.13[0.99- \\
1.29]\end{array}$ & 0.082 \\
\hline H2 & C & G & $13.79(0.01)$ & $23.74(0.01)$ & $\begin{array}{l}1.97[1.01- \\
3.84]\end{array}$ & $0.042^{*}$ \\
\hline H3 & T & A & $553.79(0.26)$ & $473.74(0.25)$ & $\begin{array}{l}0.97[0.84- \\
1.11]\end{array}$ & 0.630 \\
H4 & T & G & $108.21(0.05)$ & $48.26(0.03)$ & $\begin{array}{l}0.49[0.35- \\
0.70]\end{array}$ & $<0.001^{*}$ \\
\hline
\end{tabular}

SNP2: rs2483205; SNP4: rs562556. *: $p<0.05$.

\section{Figures}

a

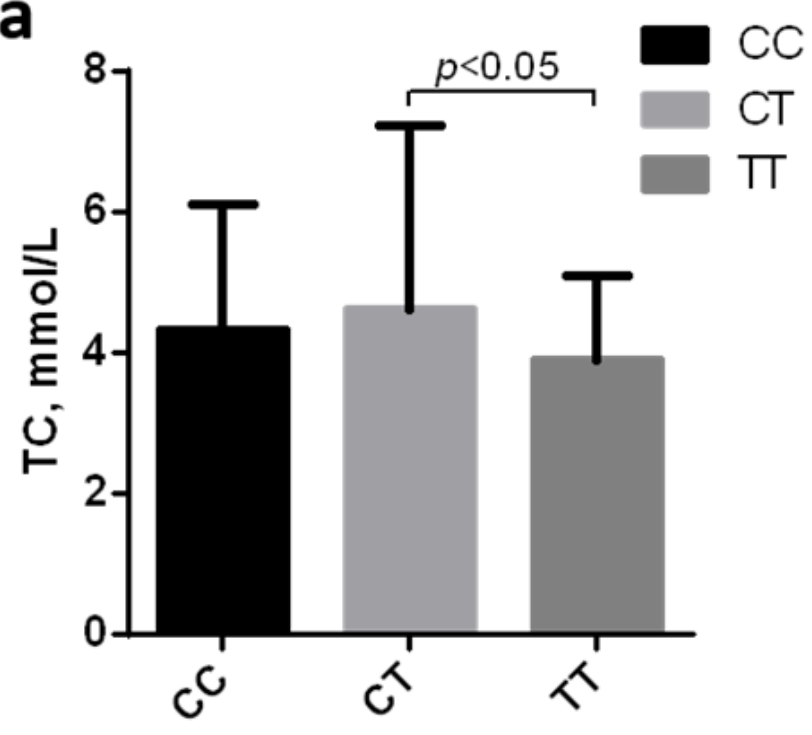

rs2483205

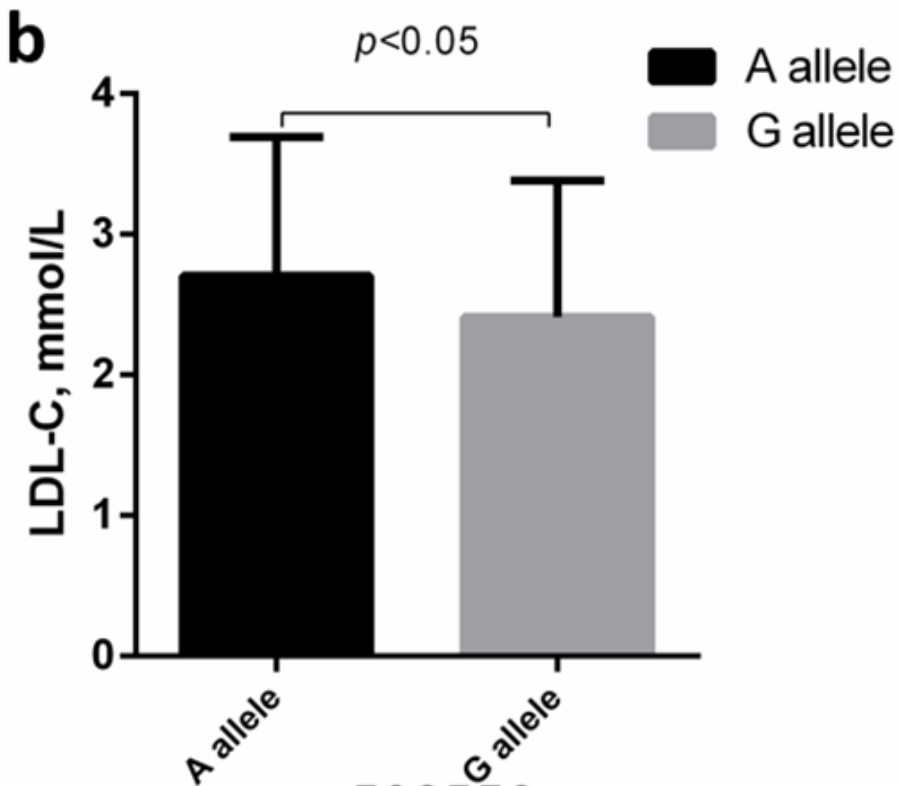

rs562556

\section{Figure 1}

TC and LDL-C levels for different genotypes of rs2483205 and rs562556, respectively. a, TC levels for different genotypes of rs2483205. Individuals with TT genotype in rs2483205 polymorphism has significantly lower TC level than CT genotype $(4.62 \pm 2.62 \mathrm{mmol} / \mathrm{L}$ vs. $3.91 \pm 1.20 \mathrm{mmol} / \mathrm{L}, p=0.025) . \mathrm{b}$, LDL-C levels for different alleles of rs562556. There is lower LDL-C level in $G$ allele than A allele in the rs562556 (2.71 $\pm 0.99 \mathrm{mmol} / \mathrm{L}$ vs. $2.41 \pm 0.97 \mathrm{mmol} / \mathrm{L}, \mathrm{p}=0.048)$. 